\title{
Effects of Air Entrainment on Fluid Transients in Pumping Systems
}

\author{
T. S. Lee, H. T. Low and D. T. Nguyen \\ Department of Mechanical Engineering, National University of Singapore, Singapore, 119260 \\ Email: mpeleets@nus.edu.sg
}

(Received February 27, 2007; accepted May 27, 2007)

\begin{abstract}
In pumping installations, fluid transient computations are necessary to achieve safety, efficiency and economy in design and operation. In some systems, where air content and air entrainment exist, such computations become highly inaccurate when constant wave speed is assumed. In this paper, a numerical model and a computational procedure have been developed to investigate the effects of air entrainment on the pressure transient in pumping systems. Free gas in the fluid and cavitation at the fluid vapour pressure were modeled in the form of variable wave speed model, which was numerically solved by the method of characteristics. This model was tested for the case of pump trips due to power failures. The pressure transient results obtained by this variable wave speed model were analyzed and compared with those results obtained by constant wave speed model and with the experimental results of other investigators.
\end{abstract}

Keywords: Pressure transient, Air entrainment, Variable wave speed

\section{NOMENCLATURE}

a wave speed

$A_{1}, A_{2}, A_{3}$ constants for pump H-Q curve

$\mathrm{B}_{1}, \mathrm{~B}_{2}, \mathrm{~B}_{3}$ constants for pump T-Q curve

$\mathrm{C}_{1}, \mathrm{C}_{2}, \mathrm{C}_{3}$ constants for pump $\eta-\mathrm{Q}$ curve

$\mathrm{C}_{\mathrm{l}} \quad$ parameter describing pipe constraint

D mean diameter of pipe

E modulus of elasticity

e local pipe wall thickness

$f \quad$ friction factor

g gravitational acceleration

$\mathrm{H} \quad$ gauge piezometric pressure head

I pump set moment of inertia

$\mathrm{k}$ time level

$\mathrm{K} \quad$ bulk modulus of elasticity

$\mathrm{N}_{\mathrm{i}}^{\mathrm{k}} \quad$ pump speed in rpm

$\mathrm{N}$ total number of node points

$\mathrm{n}_{\mathrm{p}} \quad$ number of pumps in a pumping station

$\mathrm{P} \quad$ pressure inside the pipe

Q fluid flow rate
$\mathrm{R} \quad \mathrm{C}^{+}$line intercept on $\mathrm{x}$-axis

$\mathrm{S} \quad \mathrm{C}^{-}$line intercept on $\mathrm{x}$-axis

$\mathrm{T}$ pump torque

$\mathrm{t}$ time

V flow velocity

$\mathrm{x} \quad$ distance along pipeline

$\alpha \quad$ pipeline inclination

$\eta \quad$ pump efficiency

$\Delta \mathrm{t}^{\mathrm{k}} \quad$ time step at $\mathrm{k}^{\text {th }}$ time level

$\Delta \mathrm{x} \quad$ node point distance along pipeline

$\varepsilon \quad$ fraction of gas in liquid

$\varepsilon_{0} \quad$ initial air void fraction

$\varepsilon_{\mathrm{g}} \quad$ fraction of dissolved gas in liquid

$\rho \quad$ density of fluid

\section{Subscripts}

d dissolved

e equivalent

r released

0 initial

\section{INTRODUCTION}

In pumping installation, fluid transient computation is necessary to predict excessive transient pressures which may cause collapse of pipelines, and damage of hydraulic components. In these systems, air content and air entrainment always exist and affect the pressure transient. Air in pumping systems comes from three primary sources. The first source 
of air is trapped air pockets at the top of the pipe cross-section at high points along pipe profile due to the incomplete removal of air or the progressive upward migration of air pockets. The second source of air is free gas, dissolved gas in the flow which contains approximately $2 \%$ volume of water. The third source of air comes from mechanical equipment in systems. This air may be forced into the systems as a result of falling jets of sewage into the pump sump, attached vortex formation, and adverse flow path towards operating pump. This air may also be admitted though packing, air valves, air vessel, etc. under vacuum conditions.

The effects of air entrainment on the pressure transient in pumping systems were firstly studied by Whiteman and Pearsall $(1959,1962)$ in their pump shut-down tests. In practice, analysis of pressure transient with air entrainment face many difficulties owing to lack of information such as: the location and size of air pockets in the pipelines; the amount of free air bubbles distributed in the liquid, and the physical process of release and absorption of air in the liquid. Numerical modelling of fluid transients with air entrainment has been the subject of much research. The concentrate vaporous cavity model (Brown 1968, Provoost 1976) and the air release model (Fox 1972, Wylie 1980) have been proposed in literature showing reasonable prediction of pressure transient behaviours in pipeline systems. The variable wave speed model used by Lee (1991), Borga et al (2004) can improve the simulation of experimental observations in terms of shape of the pressure peaks, the frequency of the oscillations and the rate of decay. When air is entrained such that the gas void fraction is significant and two phase motion occurs, it become necessary to introduce multiphase modeling (Huygens et al, 1998, Fujii and Akagawa, 2000 and Lee et al, 2004).

This paper aims to investigate the effects of air entrainment on pressure transient in a typical pumping system. The system consists of a lower reservoir, a group of three pumps in parallel which has a check valve in each branch, and a pipeline system discharging into an upper reservoir. The most dangerous case of pressure transient is the stop of all three pumps in the station due to a power failure. In this case, the following events take place: (i) the flow rapidly diminishes to zero and then reverses, (ii) when the flow reverses, positive pressure waves propagate downstream of the pump towards the reservoir, and negative pressure waves propagate upstream of the pipe towards the suction of the pump, (iii) the pump rapidly losses its forward rotation, and reverses, (iv) to prevent reverse flow through the pump, when the flow reverses the check valve is activated and closed. A large pressure transient occurs in the pipeline. A variable wave speed model is proposed to include the effects of free gas in the fluid and cavitation at the fluid vapour pressure on the pressure transient in the pipeline. This model is solved numerically by using the method of characteristics.

\section{VARIABLE WAVE SPEED MODEL}

In this paper, the variable wave speed model assumes the existence of free entrained air content $\varepsilon_{0}$ and dissolved gas content $\varepsilon_{g}$ in the liquid at atmospheric pressure. Some assumptions were applied in this model: (i) the gas-liquid mixture is homogeneous, (ii) free gas bubbles in the liquid follow a polytrophic compression law with $n=1$.21.3 , and (iii) the pressure within air bubbles is in equilibrium with the local fluid pressure. When the local pressure falls below the liquid vapour pressure, an amount of dissolved gas content $\alpha_{r} \varepsilon_{g}$ is assumed to release instantaneously. The local pressure is kept constant and is equal to the vapour pressure. When the local pressure recovers to a value higher than the vapour pressure, an amount of gas content $\alpha_{d} \varepsilon_{g}$ is assumed redissolved into the liquid.

The effective bulk modulus $K_{T}$ of the gas-liquid mixture, including the pipe distensibility effect and pipe constraint condition $c_{l}$ is given by (Fox 1984, Lee 1991):

$\frac{1}{K_{T}}=\frac{1}{K}+\frac{\varepsilon}{n p}+\frac{c_{l} D}{e E}$

Along the pipeline, the fraction of air content depends on the local pressure and local air volume. Therefore, in variable wave speed model, wave speed is not constant and is calculated for each point $i$ which has local pressure $p_{i}$ and air fraction content $\varepsilon_{i}$ :

$a_{i}^{k}=\left[\rho_{w}\left(1-\varepsilon_{i}^{k}\right)\left(\frac{1}{K}+\frac{\varepsilon_{i}^{k}}{n p_{i}^{k}}+\frac{c_{l} D}{e E}\right)\right]^{-1 / 2}$

The initial free air fraction $\varepsilon_{0}$ and dissolved gas fraction $\varepsilon_{g}$ must be specified. Then, the initial variable wave speed along the pipeline is computed through the absolute pressure distribution. This value is then used to calculate the pressure distribution for the next time level. The air fraction for the next time level is given by (Lee 1991):

$\varepsilon_{T}^{k+1}=\left(\frac{p_{i}^{k}}{p_{i}^{k+1}}\right)^{1 / n} \varepsilon_{i}^{k}$ and $\varepsilon_{0}^{k+1}=\left(\frac{p_{0}}{p_{i}^{k+1}}\right)^{1 / n} \varepsilon_{0}$

For $p_{i}^{k+1} \geq p_{g}$ and $\varepsilon_{T}^{k+1} \leq \varepsilon_{0}^{k+1}+\alpha_{r} \varepsilon_{g}$ : 
$\varepsilon_{i}^{k+1}=\varepsilon_{T}^{k+1}$

For $p_{i}^{k+1} \geq p_{g}$ and $\varepsilon_{T}^{k+1}>\varepsilon_{0}^{k+1}+\alpha_{r} \varepsilon_{g}$ :

$\varepsilon_{i}^{k+1}=\left(\frac{p_{i}^{k}}{p_{i}^{k+1}}\right)^{1 / n}\left(\varepsilon_{i}^{k}-\alpha_{r} \varepsilon_{g}\right)$

For $p_{i}^{k+1}<p_{g}$ :

$\varepsilon_{i}^{k+1}=\left(\frac{p_{i}^{k}}{p_{g}}\right)^{1 / n}\left(\varepsilon_{i}^{k}+\alpha_{r} \varepsilon_{g}\right)$

The new air fraction content is then substituted into Eq. (2) to compute the variable wave speed along the pipeline for the next time level.

\section{THE METHOD OF CHARACTERISTICS AND BOUNDARY CONDITIONS}

By using the method of characteristics, the governing equations for transient flow can be described by the respective $C^{+}$, and $C^{-}$ characteristic equations:

$\pm \frac{g}{a} \frac{d H}{d t}+\frac{d V}{d t} \pm \frac{g}{a} V \sin \alpha+\frac{f_{l} V|V|}{2 D}=0$

$\frac{d x}{d t}=V \pm a$

Then, the characteristic equations can be approximated by finite difference expressions:

$$
\begin{aligned}
& \frac{g}{a_{R}} \frac{H_{i}{ }^{k+1}-H_{R}}{\Delta t^{k}}+\frac{V_{i}^{k+1}-V_{R}}{\Delta t^{k}} \\
& +\frac{g}{a_{R}} V_{R} \sin \alpha_{i}+\frac{f_{l_{R}} V_{R}\left|V_{R}\right|}{2 D}=0 \\
& \frac{x_{i}-x_{R}}{\Delta t^{k}}=V_{R}+a_{R} \\
& -\frac{g}{a_{S}} \frac{H_{i}^{k+1}-H_{S}}{\Delta t^{k}}+\frac{V_{i}^{k+1}-V_{S}}{\Delta t^{k}} \\
& -\frac{g}{a_{S}} V_{S} \sin \alpha_{i}+\frac{f_{l_{S}} V_{S}\left|V_{S}\right|}{2 D}=0 \\
& \frac{x_{i}-x_{S}}{\Delta t^{k}}=V_{S}-a_{S}
\end{aligned}
$$

where $i$ denotes the regular $\mathrm{x}$-mesh point value at location $x=(i \Delta x)$ and $k$ denotes the irregular time level corresponding to the time at $t^{k}=\Sigma\left(\Delta t^{k}\right)$. Time step $\Delta t^{k}$ at each time level is determined by the Courant-Friedrichs-Levy (CFL) criterion:

$\Delta t^{k}=\min \left[k_{i} \Delta x /\left(\left|V_{i}\right|\right)+a_{i}\right]$ for $\mathrm{i}=0,1, \ldots, \mathrm{N}$

where $k_{i}$ is a constant less than 1.0.

The value of velocity and pressure head at the point of interception $R$ and $S$ can be evaluated from velocity and pressure head conditions known at $A$, $B$, and $C$ by a linear interpolation procedure. The conditions at $R$ and $S$ are then substituted into Eqs. (8)-(11) to compute the solution at the $(k+1)^{\text {th }}$ time level at point $i$.

The pump characteristics in pumping station during pump stoppage and pump run-down can be described by the homologous relationship for $n_{p}$ pumps as:

$$
\begin{aligned}
& H_{e}^{k+1}=A_{1}\left(N^{k+1}\right)^{2}+\left(A_{2} / n_{p}\right)\left(N^{k+1}\right) Q_{0}^{k+1} \\
& +\left(A_{3} / n_{p}^{2}\right)\left(Q_{0}^{k+1}\right)^{2} \\
& T_{e}^{k+1}=\left(B_{1} n_{p}\right)\left(N^{k+1}\right)^{2}+B_{2}\left(N^{k+1}\right) Q_{0}^{k+1} \\
& +\left(B_{3} / n_{p}\right)\left(Q_{0}^{k+1}\right)^{2} \\
& \eta_{e}^{k+1}=C_{1}+\left(C_{2} / n_{p}\right)\left(Q_{0}^{k+1} / N^{k+1}\right) \\
& +\left(C_{3} / n_{p}^{2}\right)\left(Q_{0}^{k+1} / N^{k+1}\right)^{2} \\
& T_{e}=-I_{e} d \omega / d t
\end{aligned}
$$

where $H_{e}^{k+1}=H_{0}{ }^{k+1}, I_{e}=n_{p} I, \omega=2 \pi N, Q$ is the flow rate, $n_{p}$ is the number of pumps, $A_{1}, A_{2}, A_{3}, B_{1}$, $B_{2}, B_{3}$, and $C_{1}, C_{2}, C_{3}$ are single pump constants. $H_{e}, T_{e}$, and $\eta_{e}$ are the equivalent pump variables.

At time $\mathrm{t}^{k}=k \Delta t, N^{k}, Q^{k}, T^{k}, H^{k}$, and $\eta^{k}$ are assumed known. For time $t^{k+1}=(k+1) \Delta t$ the computation for pump run down characteristic is given by the following procedure:

1) Estimated a new pump speed:

$N_{0}^{k+1}=N^{k}-T^{k} \frac{60 \Delta t}{2 \pi I}$

2) Estimated $H^{k+1}, Q^{k+1}$ by solving Eq. (13) together with the $C$ characteristic line Eqs. (10)(11). (14)-(15) speed:

3) Obtained $T^{k+1}$ and $\eta^{k+1}$ by solving Eqs.

4) Improved estimation of pump run-down 
$N_{1}^{k+1}=N_{0}^{k}-\left(\frac{T^{k+1}+T^{k}}{2}\right) \frac{60 . \Delta t}{2 \pi I}$

5) If $\left|N_{l}{ }^{k+1}-N_{0}{ }^{k+1}\right|$ larger epsilon (0.1RPM), set $N_{0}^{k+1}=N_{1}^{k+1}$ and repeat steps 2) to 5)

6) Else $N^{k+1}=N_{l}^{k+1}$ and obtain $Q^{k+1}, H^{k+1}$, $T^{k+1}$ and $\eta^{k+1}$ which are defined at $t^{k+1}=(k+1) \Delta t$.

When reverse flow is encountered in the pumps, check valve is assumed closed. From this instant, $V_{0}^{k+1}$ is assumed to be zero for the $C^{+}$characteristic line at $i=0$ for all subsequent time levels. The downstream boundary condition is assumed a constant head reservoir, i.e. $H_{N}{ }^{k+1}=$ constant for all time levels and this is solved with the $C^{+}$ characteristic line Eqs. (8)-(9) for $V_{N}^{k+1}$ for each time level.

\section{RESULTS AND DISCUSSION}

In this paper, the effects of air entrainment on pressure transients generated by simultaneous pump trip at pumping station were studied using a pipeline contour in Fig. 1. The pumping station uses three parallel centrifugal pumps to supply water to an upper reservoir through a $0.985 \mathrm{~m}$ diameter pipe. The value of variables downstream of pump (check valve position) is specially noticed in this paper.

Figure 2 shows the general effects of air entrainment on pressure transient in pumping system after pumps trip. For an unprotected pumping station, a study of many corresponding numerical experiments using variable wave speed model shows distinct characteristic differences of pressure surges. (i) The first pressure peak is higher than that predicted by the constant wave speed model, and the time to get the first peak as well as the time to complete a pressure surge period is longer. (ii) The damping of the pressure surges is noticeably faster in comparison with the damping in constant wave speed model. (iii) The pressure surges are asymmetric with respect to the static head, while the pressure surges for the constant wave speed model are symmetric with respect to the static head. (iv) With the variable wave speed model, the pressure transient demonstrates longer periods of down-surge and shorter periods of upsurge in comparison with the pressure transient of no air content case.

Figures 3, 4, 5 and 6 show that the pressure transient behaviour and wave speed vary significantly with the initial amount of air within the pumping system. When the initial air void fraction is increased, the pressure head of the first pressure peak grossly increases to a maximum value then slightly decreases; the pressure surge periods increase. The presence of air content in fluid greatly reduces the wave speed. The increase of initial air void fraction leads to the decrease of the wave speed. The dependence of wave speed on the initial air void fraction implies that the effects of air entrainment on pressure transient are more significant under low-pressure conditions, where its volume is greater than under high-pressure conditions. This also explains the asymmetric and different periods of down-surge and upsurge in variable wave speed model. These characteristics were also observed experimentally by previous investigators such as Whiteman and Pearsall (1959, 1962), Dawson and Fox (1983), Jonsson (1985), etc.

Previous studies have also given several explanations for above effects of air entrainment on pressure transient. For the increase in peak pressure, Jonsson attributed the increase in peak pressure to the compression of 'an isolated air pocket' in the flow. Dawson and Fox proposed the 'cumulative effect of minor flow changes during the transient'. Lee et al. (1991, 2004) explain by the lapping of the effects of two factors: (i) the delay wave reflection at reservoir, and (ii) the change of wave speed. Free air in the fluid increases the effective bulk modulus and thus lowers the average wave speed. As a result, the wave reflection at reservoir is delayed; thus a more complex variation in pressure interaction occurs in the system, culminating in a peak at a specific transient interval. Meanwhile, the reduction of the wave speed of the mixture directly causes changes in the strength of pressure oscillations. When the positive effect from the delayed wave reflection couldn't compensate for or exceed the negative effect from the reduction of wave speed, a suppressed pressure peak happens. This explains why the first peak pressure increases initially and then decreases with the increase of the initial air void fraction. Beside, in the variable wave speed model, air content is an important source of damping beside friction and minor losses. Damping effect of air content is suggested in the forms of (i) direct damping owing to the increased effective bulk viscosity of the fluid-gas mixture, (ii) losses due to slip between air bubbles and water, (iii) thermodynamic losses, and (iv) indirect damping due to partial wave reflection. This explains the fast damping of the pressure surge in the variable wave speed model in comparison with the constant wave speed model.

\section{CONCLUSIONS}

A variable wave speed model has been introduced to study the effects of air entrainment on pressure transient in pumping system following power failure and check valve closure when the flow reverses. Free gas in the liquid and cavitation at the 
vapour pressure are modeled. Numerical experiments show that where gas content and air entrainment exist, the first peak pressure is amplified; the damping effect is fast; and pressure surges are asymmetric with respect to static head. The pressure transient shows a longer period for down-surge and a shorter period for up-surge. The paper also introduced some explanations for above effects of air entrainment on pressure transient behaviours of pumping systems. However, the results still remained some doubts such as the precise physical cause of large surge damping, the physical process of gaseous diffusion in a closed conduit subjected to unsteady flow, etc. This limitation is partly due to the random nature of bubble nucleation, coalescence and growth in flow fields. Therefore, much more research in this field is needed.

\section{REFERENCES}

Borga, A., Ramos, H., Covas, D., Dudlick, A. and Neuhaus, T. (2004), Dynamic effects of transient flows with cavitation in pipe systems, Proceedings of the $9^{\text {th }}$ International Conference on Pressure Surges - The practical application of surge analysis for design and operation, British Hydromechanics Research Group (BHRG), Chester, UK, pp. 605-617.

Thorley, A. R. D. (2004), Fluid transients in pipeline systems: a guide to the control and suppression of fluid transients in liquids in closed conduits, Professional Engineering, London, second edition.

T.S Lee, H. T. Low and W. D. Huang (2004), 'Numerical study of fluid transient in pipes with air entrainment', International Journal of Computational Fluid Dynamics, Vol. 18 (5), pp. 381-391.

Fujii, T. and Akagawa, K. (2000), 'A study of water hammer phenomena in a one-component two-phase bubbly flow', JSME Int Journal Series B-Fluids and Thermal Engineering, Vol 43, Pt 3, pp. 386-392.

Huygens, M., Verhoeven, R. and Van Pocke, L. (1998), 'Air entrainment in water hammer phenomena', Advances in Fluid Mechanics II, Vol 21, pp. 273-282

E. B. Wylie and V. L. Streeter (1993), Fluids Transients in Systems, Prentice Hall, Englewood Cliffs, NJ 07632.
T. S. Lee (1991), 'Numerical computation of fluids pressure transient in pumping installations with air entrainment', International journal for numerical methods in fluids, Vol. 12, pp. 747-763.

L. Jonsson (1985), 'Maximum transient pressures in a conduit with check valve and air entrainment', Proc. Int. Conf. on the Hydraulics of pumping stations, Manchester, BHRA, Cranfield, pp. 55-76.

J. A. Fox (1984), Hydraulic analysis of unsteady flow in pipe network, Macmillan, London

P. A. Dawson and J. A. Fox (1983), 'Surge pressures at Riding Mill pumping station: actual values and theoretical predictions', Proc. $4^{\text {th }}$ Int. Conf. on Pressure Surges, Bath, BHRA, Cranfield, pp. 427-445.

E. B. Wylie (1980), 'Free air in liquid transient flow', Proc. $3^{\text {rd }}$ Int. Conf. on Pressure surges, Canterbury, BHRA, Cranfield, pp. 12-23.

E. B. Wylie and V. L. Streeter (1978), Fluids Transients, McGraw-Hill, New York

G. A. Provoost (1976), 'Investigation into cavitation in a prototype pipeline caused by waterhammer', Proc. $2^{\text {nd }}$ Int. Conf. on Pressures, London, HBRA, Cranfield, pp. 35-43.

J. A. Fox (1972), 'Pressure transients in pipe networks - a computer solution', Proc. $1^{\text {st }}$ Int. Conf. on Pressure Surges, Canterbury, BHRA, Cranfield, pp. 68-75.

R. J. Brown (1968), 'Water-column separation at two pumping plants', J. Basic Eng., ASME, 521531.

I. S. Pearsall (1965/1966), 'The velocity of waterhammer waves', Proc. Inst. Mech. Eng., 180, pt. 3E, 12-20.

K. J. Whiteman and I.S. Pearsall (1962), 'Reflux valve and surge tests at a station', Fluid Handling, XIII, 248-250, 282-286.

K. J. Whiteman and I. S. Pearsall (1959), 'Reflux valve and surge tests at Kingston pumping station’, Brit. Hydromech. Res. Assoc./National Engineering Laboratory Joint report 1. 
T.S. Lee et al. / JAFM , Vol. 1, No. 1, pp. 55-61, 2008.

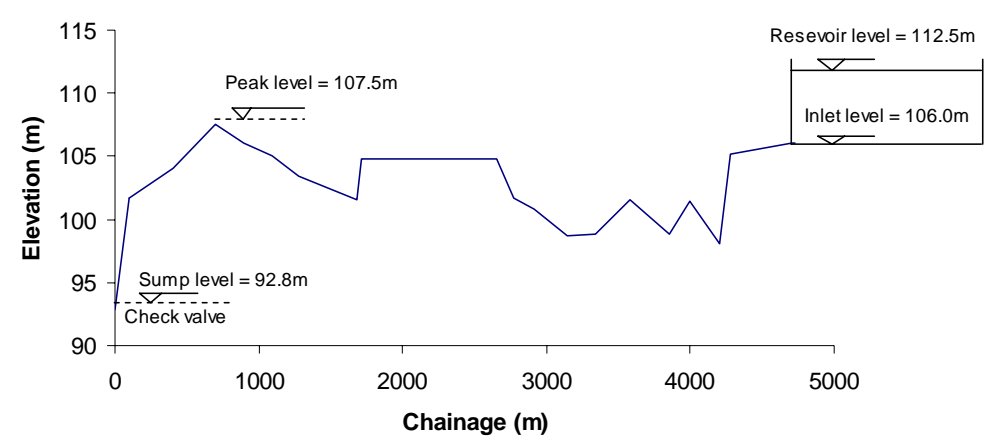

Fig. 1- Pumping station pipeline profile.

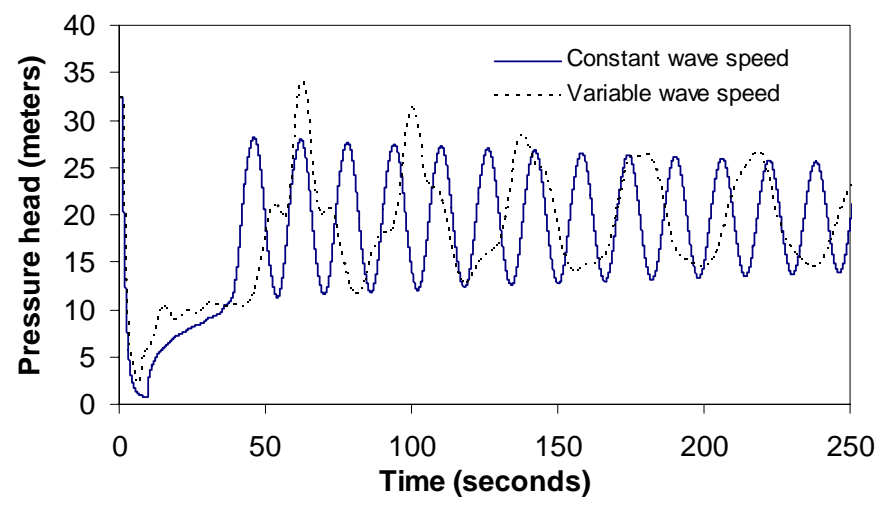

Fig. 2- Pressure head downstream of pump

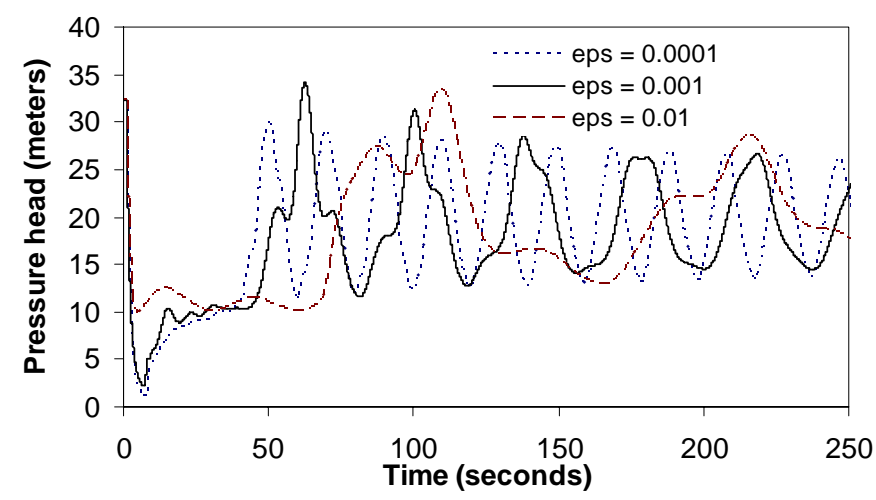

Fig. 3- Pressure head downstream of pump with different initial air void fraction

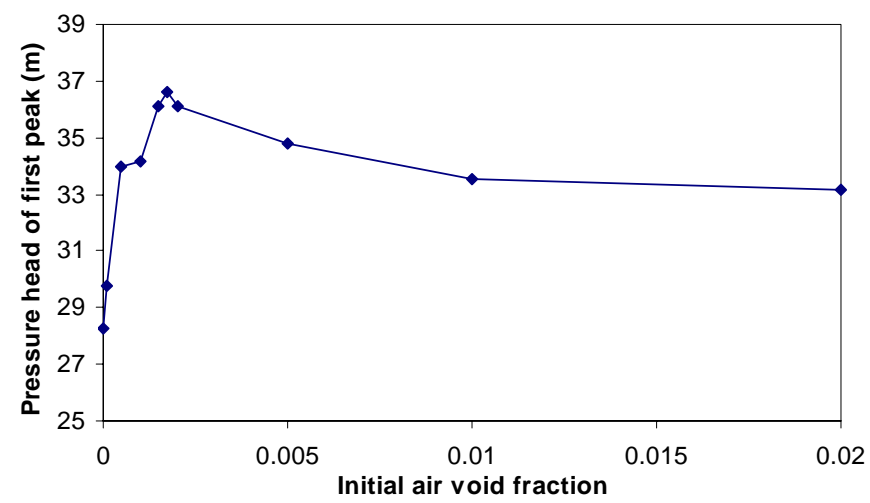

Fig. 4- Pressure head of first pressure peak with initial air void fraction 
T.S. Lee et al. / JAFM , Vol. 1, No. 1, pp. 55-61, 2008.

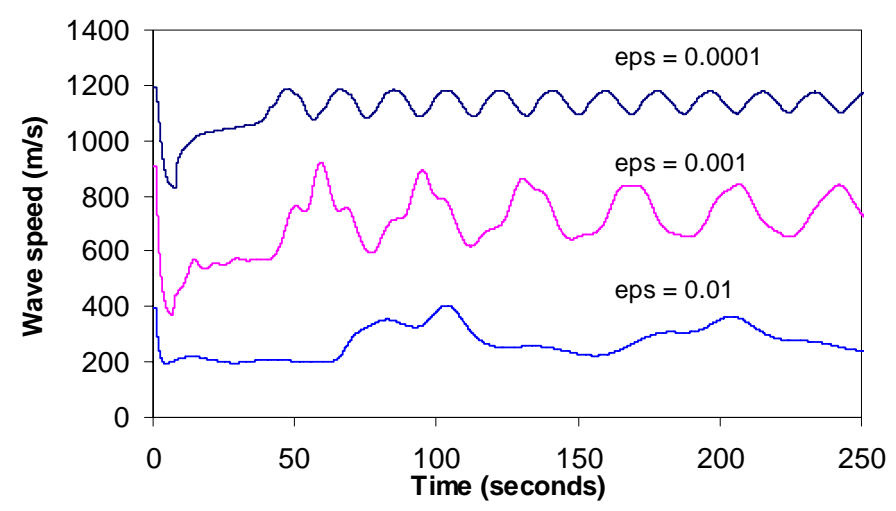

Fig. 5- Wave speed downstream of pump with different initial air void fraction

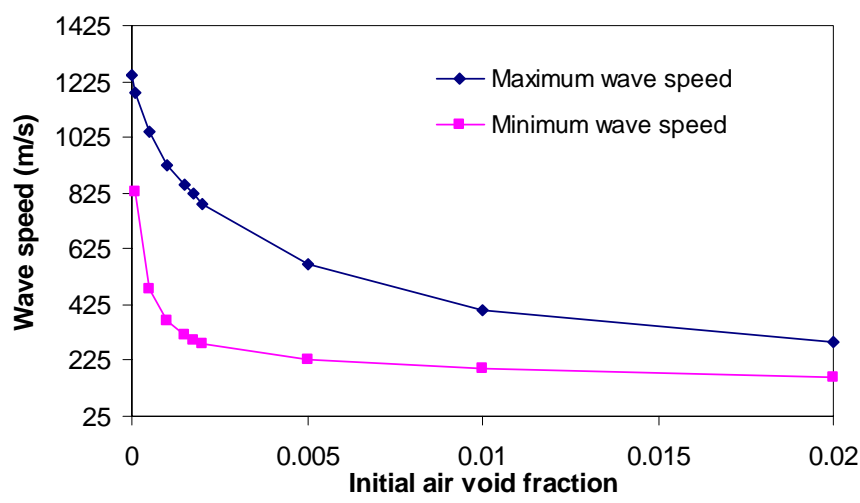

Fig. 6- Wave speed downstream of pump varies with initial air void fraction 